\title{
An Optimized Technique for Sharing Air Quality Index Using Cloud Technology
}

\author{
N. Vallileka, S.Jai Ganesh, Nimmathi Satheesh, R.SivaKumar, N.Muthu Lakshmi
}

\begin{abstract}
This paper studies the sharing the messages of Air Quality Index (AQI) in Metropolitan areas. AQI specifies the percentage of pollutants mixed in air which creates problem in health. The pollutants level in the AQI values is between 0 to 500. Air pollution sensors are used to monitor the air pollution for outdoor environments and the cloud technology is used to display to public via private cloud. Prototype was developed with the aim to create awareness and public engagement in restoring the environment back to its healthy state. Using the prototype, the users can interact with the environment sensors in the field of view to access and visualize latest and historic environment measurements.
\end{abstract}

Keywords : AQI, Air Quality index, mobile Communication, cloud with AQI, air pollution sensors.

\section{INTRODUCTION}

There has been a drastic change in air pollution since the start of industrial revolution. Though industries are seen as growth propellers the impact it creates in the environment is not healthy. To add to the woes, vehicles used for transportation and fertilizers used in agriculture contribute to pollution too. People are aware of the ill-effects of pollution.

However, people do not monitor or track it every day. Air pollution can be due to natural cause or due to abnormal. The highest pollutants level makes pollution in the natural air creates fire accidents in the forest, dust-storms, pollution, and etc. Some of the human-made pollution are wood cutting, coal mining and fuels-based pollution. In order to measure the pollution level AQI is used where it displays the information about the major pollutants mixed in the air. It can be measured at particular geo-location without any infrastructure development.

Monitoring the air quality helps to obtain the purity of the air based on the mixed content's information. It helps to inform the public about the air pollution since they can take action for breathing air. Because polluted air makes lot of serious health issues to the human. It creates lung, brain and breathing issues to the public and put them in critical situation. Identifying the air quality helps to save people from the health issues and make the people reduce their activities related to pollutants. Once people reduce the pollutants-based activities it decreases the pollutants level mixed in the air. To do that, an alarm is installed/deployed in the target geo-location where it makes an alarm whenever the pollutants level exceeds their limit. In order to do that,

Revised Manuscript Received on January 2, 2020.

N. Vallileka, Dept. of computer Applications, PSNACET, Dindigul, Tamilnadu, India

S.Jai Ganesh, Dept. of computer Applications, PSNACET, Dindigul, Tamilnadu, India

Nimmathi Satheesh, Dept. of computer Applications, PSNACET, Dindigul, Tamilnadu, India

R.SivaKumar, Dept. of Chemistry, PSNACET, Dindigul, Tamilndau, India

N.Muthu Lakshmi, TCS, Bangalore, Karnataka, India this paper proposed an IoT based cloud application like android application. When the pollutant level increases then an alarm is generated in the android mobiles connected in the cloud network (within the application). Hence based on the pollution the public informed by alarm to get remedy to avoid health issues. The proposed system identifies the level of N2, NO2, SO2, and P.M10 using various kinds of sensors installed in the network. It also identifies temperature and humidity by relevant sensors connected in wireless communication medium. The android application is designed and installed in the particular place where it is required really. Each user needs to register in the application using their mobile device. The sensors observe the data along with the AQI and transmit to the application server and the intimation to the mobile.

\section{AIR POLLUTION INDEX BACKGROUND}

The air pollution is calculated in terms of AQI, where its range of values is considered from 0 to 500 and it is given in detail in Table-2.1.

One of the main forms of air-pollution is created by transportation and fuel usage applications. High level of pollution makes acid rain which affects the drinking water, soil, buildings, vegetation and destroy the aquatic life [17].

Table 2.1 Air quality Index

\begin{tabular}{|c|c|}
\hline Air Quality & Air Quality Index \\
\hline Good & $0-50$ \\
\hline Moderate & $51-100$ \\
\hline $\begin{array}{c}\text { Unhealthy for } \\
\text { Sensitive Groups }\end{array}$ & $101-150$ \\
\hline Unhealthy & $151-200$ \\
\hline Unhealthy (Alert) & m $201-300$ \\
\hline
\end{tabular}

\section{PROPOSED TECHNIQUES}

\subsection{Air Pollution Sensors}

Some kind of sensors are used as devices for monitoring and calculating air pollution. They may be used for indoor and outdoor environments. Even though there are different types of sensors for air pollution, the main are five components: 
- ozone,

- particulate matter,

- carbon monoxide,

- sulfur dioxide, and

- nitrous oxide.

Initially the cost of the sensors is high, now with technological advancements these sensors cost is low and widespread throughout the population. By this project the sensors are fitted in the main areas for Metropolitan cities to maintain storage pool to store the monitored data using the air quality system. It stores all the monitored data gathered from the particular geolocation where the system installed. This application is used in top most metropolitan cities.

By using these kinds of sensor, all the possible measures related pollutants are collected as a spatiotemporal data where it lists out all the chemical information which affects the human in and around a specific location. Also, it mainly says about the air pollution [4]. The evidence of the air pollution happened in the metropolitan and industrial cities are increased by scientific reports. The amount of death due to air pollution is increasing up to 7 million child death throughout the world in each year[8]. The pollutants are inhaled by the human and it is deposited in various parts of the body like lung, stomach and etc., which creates serious diseases like cancer and heart attacks. It can be measured by the body mass index values and blood tests. The pollutants are travelling throughout the human body and affects the vital organs of the body [9].

\subsection{Cloud Computing Techniques}

One of the highly demanding environments used to share the computer resources is cloud computing. Those devices are used to do various tasks especially data storage and computing devices. some of the famous cloud providers are Google cloud platform, iCloud, cloud foundry, Microsoft Azure, Amazon etc. The cloud clients are web browser, mobile app and terminal emulator. Three standard models are IaaS, SasS and platform as a service PasS. Here we are going to use SasS to see the AQI from cloud to mobile. Now Mobile backend as a service (BaaS) is there where web application developers and mobile application designers are link applications with cloud storage and cloud computing services with their mobile application. In cloud, users are managed. It also supports server less computing.

\section{METHODLOGY}

\subsection{Selection of Sensors}

The Pollution Sensors and their usages are:

Nox is identified by MQ6 Sensor

\section{So2 is identified by MQ6 Sensor and}

\section{P.M.10 is identified by Dust Sensors as DSM501.}

Two eco-friendly Sensors are used for temperature DHT 11 and for humidity - DHT 11

\subsection{Cloud Provider and services}

Cloud computing manage the sensors where it will coordinate between the Sensor owner, the cloud provider and the end user i.e public. The sensor owners develop the system and develop functionality to publish the data on the cloud. The cloud providers then provide the cloud services and may charge the end user with some fee for the access. We utilize the PaaS (Platform As A Service). PaaS provides a platform for software creation. Another important factor of $\mathrm{PaaS}$ is that it allows business to design and create applications that are built into the PaaS with special software components. The "Breathe-safe" application that we developed on PaaS platform utilizes various computation algorithms and AQI calculation based on current sensors values and showing it to user on GUI using JavaScript based charts \& Graph API.

This supports server less computing so virtual machines are needed to service the request based on hour basis. There are three types of deployment model private cloud, public cloud and hybrid cloud. In this paper we are using private cloud to share AQI.

\section{DESIGN CONSIDERATION AND WORKING PRINCIPLE}

Measurements of air pollutants such as Carbon monoxide (CO), Nitric Oxide (NO), Nitrogen dioxide (NO2) have been recorded since the installation[17]. Also, measurements of temperature, humidity and sound level is also made to get more information about the environment. Once the database server was set up, the content necessary for visualization was created. The contents were converted into file formats and sizes supported by SDK rendering engine and stored in the database of the server in cloud. Media content such as voice alerts or audio messages were created for real time response to the users and stored in web server in cloud Images and icons to be displayed were also created and stored.

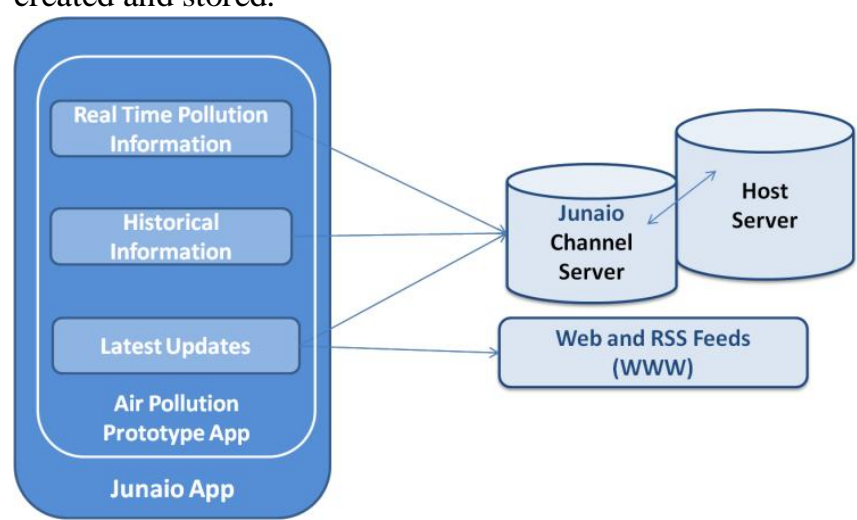

Fig5.1. Modules in Junaio App

The above figure 5.1 explains the development of such a transparent platform will act as a basis for actions towards environment protection. The structure of the methodology is as follows, Design and develop a prototype to display air pollution in the environment in the city. Evaluate mobile app prototype developed for displaying air pollution. 
In order to understand about the practical applicability of the prototype application and user perception, user evaluation of the system was conducted in comparison with an existing web application tool for monitoring air pollution in the environment. Factors of context immersion are considered for user evaluation to evaluate the immersion experience created by mobile application. A mobile application (in short mobile app) is a customized application developed using the native programming language Android that supports the platform of the smart phone device or tablet. The advantage and disadvantage of AQI displaying systems was studied using human understanding and response to the features of the application for monitoring pollution in the environment.

Taking this as the context and interest of the users, two modules were designed based on the timeline the information belongs to real-time visualization and historical data visualization. Third module is to get latest information from the research community which can be latest discovery or results from forecast modeling of environment research. The third module serves as the information portal which shares and showcases the status and results of environment researches. Three modules were designed to provide user with option to select the data they would like to visualize. These three modules constitute the layout of the prototype of air pollution visualization. The operations that should be performed on loading each module will be explained in the prototype development section. During the design of the prototype, it is important to consider some best practice design considerations such as performance, modularity, robustness, security and scalability. A well designed module should take less time to load and perform its desired action showing reliable performance during usage. It should be able to work independently with modularity and should respond in stress conditions with prompt user response. The modules should allow easy extendibility. Prototype design should accommodate the user interaction through the touch inputs and visualization of data should be designed to fit small screen of mobile phones. The visualization in the design includes text annotations, charts, and map of the location. Junaio is an Augmented Reality browser designed for $3 \mathrm{G}$ and $4 \mathrm{G}$ mobile.

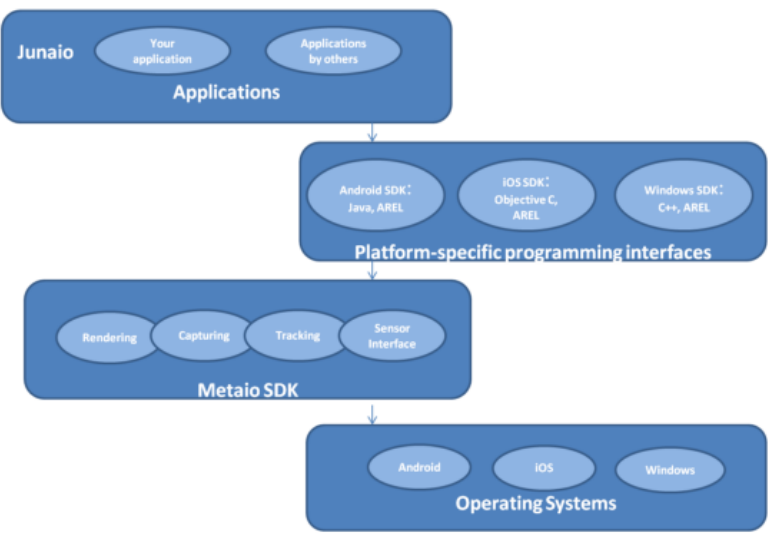

Fig:5.1 Product Architecture of Mobile App

The above figure Fig 5.1 describes the protocol architecture of Mobile App which is used for displaying the AQI.

\section{PROBLEM FORMULATION}

\subsection{Computing the AQI}

The air quality index for pollutant concentration.

The equation used to convert from concentration to AQI is :

$I=\frac{I_{\text {high }}-I_{\text {low }}}{C_{\text {high }}-C_{\text {low }}}\left(C-C_{\text {low }}\right)+I_{\text {low }}$

where:

$I=$ the (Air Quality) index,

$C=$ the pollutant concentration,

$C_{\text {low }}=$ the concentration breakpoint that is $\leq C$,

$C_{\text {high }}=$ the concentration breakpoint that is $\geq C$,

$I_{\text {low } w}=$ the index breakpoint corresponding to $C_{\text {low }}$,

$I_{\text {high }}=$ the index breakpoint corresponding to $C_{\text {high }}$. EPA's table of breakpoints is:

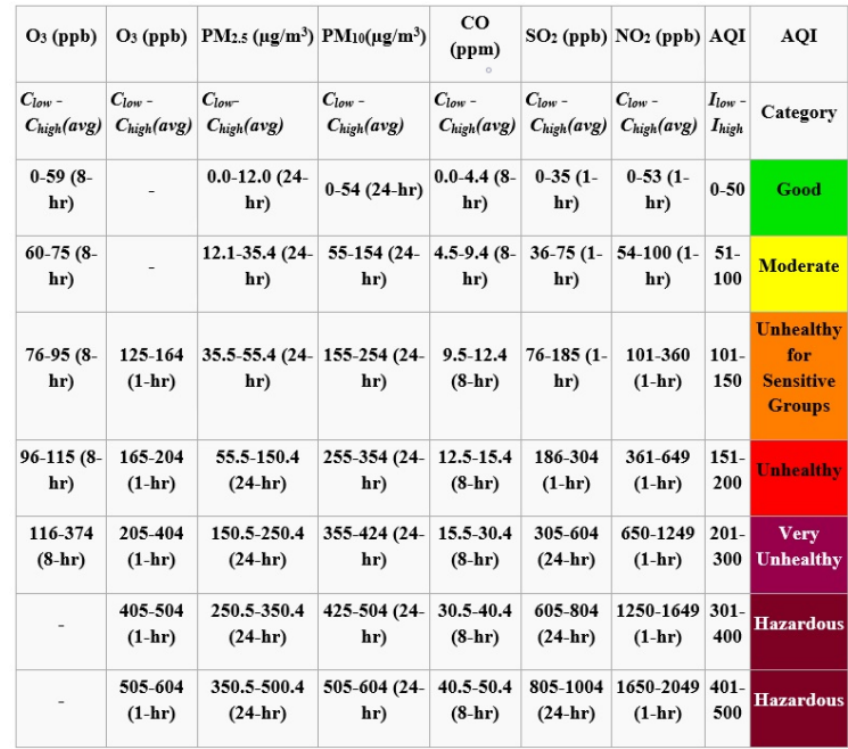

Suppose a monitor records a 24-hour average fine particle $\left(\mathrm{PM}_{2.5}\right)$ concentration of 12.0 micrograms per cubic meter. The equation above results in an AQI of:

$$
\frac{50-0}{12.0-0}(12.0-0)+0=50
$$

Corresponding to air quality in the "Good" range. To convert an air pollutant concentration to an AQI, EPA has developed a calculator.

If multiple pollutants are measured at a monitoring site, then the largest or "dominant" AQI value is reported for the location. The ozone AQI between 100 and 300 is computed by selecting the larger of the AQI calculated with a 1-hour ozone value and the AQI computed with the 8-hour ozone value. 
8-hour ozone averages do not define AQI values greater than 300; AQI values of 301 or greater are calculated with 1-hour ozone concentrations. 1-hour SO2 values do not define higher AQI values greater than 200. AQI values of 201 or greater are calculated with 24-hour SO2 concentrations.

\subsection{Prototype Testing and Publishing}

Prototype testing was carried out in the target platforms of mobile devices. Prototype was

Published in Junaio channel server as non-public for testing. Prototype validation was performed by Junaio server and the response from the server was verified. After publishing, the prototype was tested in Android and iOS platforms using mobile devices built with those platforms. iPhone $4 \mathrm{~S}$ and iPad 2 with iOS version 6.1.2 was used for testing in iOS environment. Any defects found during the testing was corrected and tested again in both operating systems. Once there were no defects detected, the prototype was published as public channel. The channel can

then be viewed by anyone who has Junaio application installed. Once the channel is published to

public, the channel can be found using the channel name or associated keywords of the channel in the Junaio directory.

\section{RESULTS}

\subsection{Air Pollution Visualization}

The channel for air pollution visualization was published as non public channel during development and testing. The quality of air in the location of metropolitan area was visualized. Figure 5.1shows augmented view of the environment sensors which measures air quality in the study area.

The distance to the each sensor and the sensor number is shown in the label of the sensors. On

navigation towards the sensors, individual sensors were located.

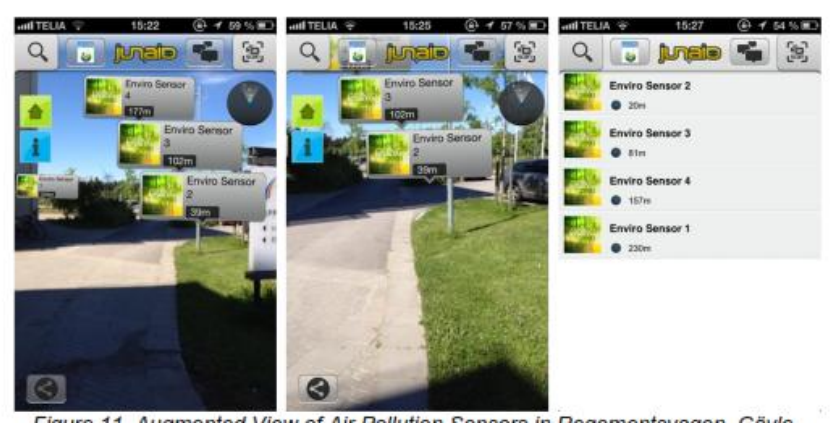

Fig: 5.1 Displaying Sensor areas

\section{CONCLUSION}

However, the proposed system work well in sharing of AQI in metropolitan areas such as Delhi, as of now which is the latest metropolitan city highly affected by air pollution. Methods like Air Quality Management Plan ,Development of new technology- electric cars, cleaner fuels, low nitrogen oxide boilers and water healers, zero polluting paints, less polluting BBQ lighter fluids, Use natural gas, Carpooling will reduce the air pollution. To provide value added services like audio/video sharing etc., are also implemented here.

\section{REFERENCES}

1. Bernstein JA, Alexis N, Barnes C, Bernstein IL, Bernstein JA, Nel A, Peden D, Diaz-Sanchez D, Tarlo SM, Williams PB: Health Effects of Air Pollution. Journal of Allergy and Clinical Immunology 2004, 114(5):1116-1123.

2. Asaad M. J. Al-Hindawi, Ibraheem Talib, "Experimentally Evaluation of GPS/GSM Based System Design", Journal of Electronic Systems Volume 2 Number 2 June 2012

3. B.Abinayaa, G.Kiruthikamani, B.Saranya, R.Gayathri, "An Intelligent Monitoring Device for sthmatics using Arduino", International Journal of Advanced Research in Electrical, Electronics and Instrumentation Engineering, ISSN (Print): 2320 3765, ISSN (Online): 2278 - 8875 Vol. 5, Issue 7, July 2016.

4. Saranya.B, Sasikala.N, Sharmila.K, Nandini.S, "An Embedded based Robotic Arm to Find Unauthenticated Code," International Journal of Engineering and Management Research Page Number: 185-190 ISSN (ONLINE): 2250-0758, ISSN (PRINT): 2394-6962, Volume-5, Issue-1, February-2015.

5. K., Thurston, G.D., Silverman, R.A., 2007. Characterization of PM2.5, gaseous pollutants, and meteorological interactions in the context of time-series health effects models. J. Expo. Sci. Environ. Epidemiol. 17 (Suppl. 2), S45eS60

6. State Environmental Protection Agency of China, "China National Ambient Air Quality Standard (in Chinese)," GB3095-2012, State Environmental Protection Agency of China, Beijing, 2012

7. CPCB, "National Ambient Air Quality Standards," 2009. http://www.cpcb.nic.in/National_Ambient_Air_Quality_S tandards.php

8. BIS, "Indian Standard Methods for Measurement of Air Pollution: Part IV-Suspended Matter," Bureau of Indian Standards, New Delhi, 1974, 11 p.

9. BIS, "Indian Standard Methods for Measurement of Air Pollution: Part VI-Nitrogen Oxides," Bureau of Indian Standards, New Delhi, 1975, $6 \mathrm{p}$.

10. BIS, "Indian Standard Methods for Measurement of Air Pollution: Part II-Sulphur Dioxide," Bureau of Indian Standards, New Delhi, $1969,11 \mathrm{p}$

11. Venkatesh, Indra. A and Murali. R, "Vehicular Ad hoc Networks (VANETs): Issues and

12. Applications", Journal of Analysis and computation, Vol. 8, No. 1 2012, pp.31-46.

13. Andrei Furda et al, "Enabling Safe autonomous driving in real-world city traffic using multiple criteria decision making”, IEEE Intelligent Transportation System Magazine, SPRING 2011, pp. 4-17.

14. S S Manvi and M S Kakkasageri, "Issues in Mobile Ad hoc Networks for Vehicular Communication",IETE Technical Review, Vol. 25, No 2 MarchApril 2008.

15. Alexandru Dorin et al, "MAC Protocols for VANETs - A Survey and Qualitative Analysis", Albert-Ludwigs-University Freiburg, seminar paper, February 2010.

16. V.Ramya, B. Palaniappan, K. Karthick, "Embedded Controller for Vehicle In-Front Obstacle Detection and Cabin Safety Alert System", International Journal of Computer Science \& Information Technrology (IJCSIT) Vol 4, April 2012

17. Mrs.N.Vallileka,Mrs.T.Kamalavalli,Dr.A.Vanitha katherine,Mrs.S.Vinothini," An optimized technique for automobile data communication to share air qulaity index ",International Journal of Mechanical Volume 9, Issue 11, November

\section{AUTHORS PROFILE}

N. Vallileka, Dept. of computer Applications, PSNACET, Dindigul, Tamilnadu, India.

S.Jai Ganesh, Dept. of computer Applications, PSNACET, Dindigul, Tamilnadu, India.

Nimmathi Satheesh, Dept. of computer Applications, PSNACET, Dindigul, Tamilnadu, India.

R.SivaKumar, Dept. of Chemistry, PSNACET, Dindigul, Tamilndau, India.

N.Muthu Lakshmi, TCS, Bangalore, Karnataka, India 